\title{
Effect of Male Diet on Accessory Gland Protein and Sperm Traits in D.melanogaster
}

\author{
Krishnan Anitha, Mysore Siddaiah Krishna* \\ Drosophila Stock Centre, Department of Studies in Zoology, University of Mysore, Manasagangothri, Mysore- 560006. Karnataka, India
}

Received July 4, 2020; Revised August 25, 2020; Accepted September 11, 2020

Cite This Paper in the following Citation Styles

(a): [1] Krishnan Anitha, Mysore Siddaiah Krishna, "Effect of Male Diet on Accessory Gland Protein and Sperm Traits in D.melanogaster," Advances in Zoology and Botany, Vol. 8, No. 6, pp. 506-511, 2020. DOI: 10.13189/azb.2020.080604.

(b): Krishnan Anitha, Mysore Siddaiah Krishna , (2020). Effect of Male Diet on Accessory Gland Protein and Sperm Traits in D.melanogaster. Advances in Zoology and Botany, 8(6), 506-511. DOI: 10.13189/azb.2020.080604.

Copyright $@ 2020$ by authors, all rights reserved. Authors agree that this article remains permanently open access under the terms of the Creative Commons Attribution License 4.0 International License

\begin{abstract}
The quality and quantity of nutrients present in the food consumed by organisms have a strong influence on reproductive fitness. The diet of individuals is apparently a key factor in reproduction with effects on their sexual performance and progeny production. Variation in reproductive success of individuals in a population depends on the quality and quantity of nutrients available and also consumed by the individuals. The investigations on the effects of diet on life events in insects include mate choice, mating incidence, activity levels, the quantity of seminal fluid and /or accessory gland proteins produced and sperm production. Here we investigate the effect of male nutrition on the accessory gland and quantity of sperms transferred to the mated female during copulation to understand its effect of male reproductive success in Drosophila melanogaster. We raised larvae on either the protein-rich diet or carbohydrate-rich diet. We found that flies consuming protein-rich diet had numerous small main cells in their accessory gland and formed a larger amount of accessory gland protein, whereas males fed on the carbohydrate-rich diet had fewer large main cells in their accessory gland and produced a significantly small amount of accessory gland proteins. Further males reared on the protein-rich diet had copulated longer and transferred a larger amount of accessory gland protein and sperms to mated females than those males which fed with wheat cream agar media or male fed on the carbohydrate-rich diet.
\end{abstract}

Keywords Drosophila Melanogaster, Reproductive Success, Male Diet, Accessory Gland, Copulation Duration

\section{Introduction}

The majority of compelling studies on accessory gland development in male Drosophila revealed that the cells in the genital imaginal disc grows in different arrangements [1]. Its fine structure depends on its stage of development and the nature of the secretion produced [2]. These glands are functional in adult and its secretion is concerned in numerous mechanisms linked to reproduction [3]. During copulation, the discharge of gland and sperm are moved to the female so these can influence male reproductive success in many ways in the mated female[4]. Structure and amount of Acp differ between species [4] and also between strains of the same species[2-5]. Its fine structure also depends on their stage of development and the nature of the secretion produced. Both intrinsic and extrinsic factors are also known to affect its structure and function [3].

While studying in various strains of Drosophila nasuta and Drosophila melanogaster, also indicate the positive relationship between the size of male and amount of accessory gland proteins [6]. In Drosophila species, it has noticed that larger size males had transmitted the higher amount of accessory gland secretion to the copulated females when compared to tiny size males. The copulating behavior of males and females [7], the outcome of copulating on immunity [8], sperm competition, its result on male reproductive fitness [9] have likewise known to influence on the amount of accessory gland discharge which is moved to mated females in species of Drosophila

Recently male age influence on accessory and sperm traits has also been studied in a few species of Drosophila. Male age shows that the major influence on size and number of the main cells in the accessory gland as a result amount of accessory 
gland protein produced varies across age. In D.melanogaster amount of accessory gland protein decreased with increasing male age along with sperm qualities [10] whereas in D.bipectinata and D.malerkotliana [11] the quantity of accessory gland protein increased with increasing male age. They also found that amount of accessory gland transferred during the act of copulation also vary across male age classes, young males of D.melanogaster shows a large amount of accessory gland protein was transmitted to the copulated females than mid-aged and older males, whereas in D.bipectinata, older males significantly transfer a larger amount of Accessory gland protein and sperms to mated females than mid-aged or young males.

Nutrition is a significant factor which is known to a huge impact on the reproduction, survival, and health of an organism. Files of D.melanogaster they feed on a diet containing carbohydrate and protein. However, the availability and quantity of carbohydrate and protein in the diet has a significant effect on growth, survival, reproduction, and health. Even though, recent investigation has been taken up in D.melanogaster to survey the male diet interconnected changes on growth, structure and function of sperm traits and accessory gland by the subsequent objectives:

1) Either or not the amount of accessory gland production varies within the various male diet routine program. Therefore, what are the relation between size, a numeral of main cells, and the amount of accessory gland proteins?

2) To check the suggestion of the connection between male diet, copulation period and the amount of the accessory gland secretion/sperm transferred to a mated female.

\section{Materials and Methods}

Flies of Oregon-K (D.melanogaster) received from the stock center of Drosophila, Zoology Department, Mysore University, be used in the present experiment. Twenty males and females were placed into Drosophila culture bottle containing wheat cream agar media and kept up at $22^{\circ} \mathrm{C} \pm 1{ }^{\circ} \mathrm{C}$ with a relative humidity of $70 \%$ utilizing $12 \mathrm{~h}$ : $12 \mathrm{~h}$ light: dark cycle. Eggs were taken from these flies using the methodology of the Delcour procedure [12]. 25 Eggs were then transferred into independent culture bottles containing $7 \mathrm{ml}$ of wheat cream agar medium (Normal culture medium; NM)/wheat cream agar medium $+60 \%$ casein (protein enrich medium; PR) and wheat cream agar media $+20 \%$ sucrose (Carbohydrate enriched media; CR). Mixing wheat cream agar media and sucrose to prepare the carbohydrate enriched medium (CR) in the ratio 4:1 (20\% carbohydrate), before adding water. Similarly, to prepare protein-enriched medium, Casein and wheat cream agar medium was mixed in the ratio $3: 2$ ratio. $7 \mathrm{ml}$ of protein-rich and carbohydrate-rich media were taken in Drosophila cultural vials and were used to culture the experimental flies in Drosophila culture vials containing PR/CR media. All vials (Drosophila culture vials) were pasted with a dried yeast solution. These culture vials were kept up in the previously mentioned lab conditions. Unmated males and females were col- lected in 3 hours of eclosion also matured them for 5-6 days. For experiments reason, these flies were utilized.

\subsection{Connection among male diet, accessory gland size, numeral of cells (per lobe), and cell size in accessory gland}

Glands of unmated male flies developed from different dietary mediums (NM, CR, PR) these glands were dissected separately using Medium A [13] also be set in $1 \mathrm{~N} \mathrm{HCl}$ for 5 min. Accessory glands photos were captured at $40 \mathrm{X}$ with the support of digital cameras. The size and shapes of the accessory gland were calculated individually following the methodology of Abolhasan and Krishna [10]. In D.melanogaster, the shape of the accessory gland was 's', 'c', or 'j' formed gland, to measure these regions of accessory gland the entire region of each accessory gland was separated into a tiny region containing triangles, trapeziums, and rectangles (Figure 1). The regular structure region was calculated separately following the procedure of Ram and Ramesh [5].

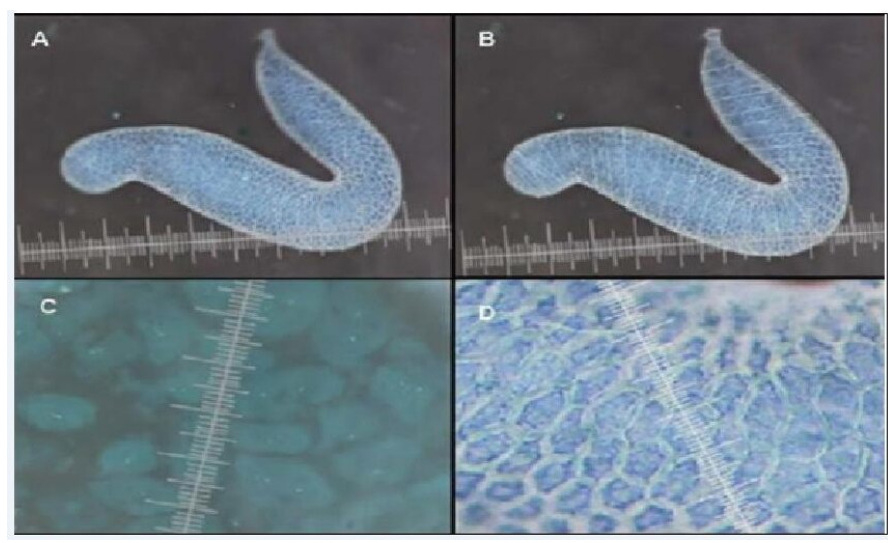

Figure 1. Total measurement is taken to know the number and size of the cell also gland size are measured in D.melanogaster flies: A.Accessory gland lobe; B. Accessory gland lobe are marked to found the accessory gland size; $\mathrm{C}$ and D. Marked accessory gland lobe for measuring the size of the gland; $\mathrm{C}$ and D.Measurement of cell size of accessory gland lobe. Excellent quality picture information is accessible on the internet.

Later capturing the photos of the dimension of accessory gland size, immediately these glands were stained using lactoaceto orcein $2 \%$ and stained up to 20 minutes, then squash these glands with $45 \%$ acetic acid and sealed using glass slide and coverslip. The main cell number in every gland was noted and tallied at 40x zoom using a counter called tally. Following this, the same glands were utilized to determine the main cell size starting with one end to another end in a polygonal cell using a micrometer at 40x zoom. Fifty replicates from each the three dietary medium was used to calculate the size of the accessory gland and numeral of the main cell and size of the accessory gland of males (NM, CR, and PR). One-way Anova was done on the above information utilizing Statistical Product and Service Solutions- 20 programs. 


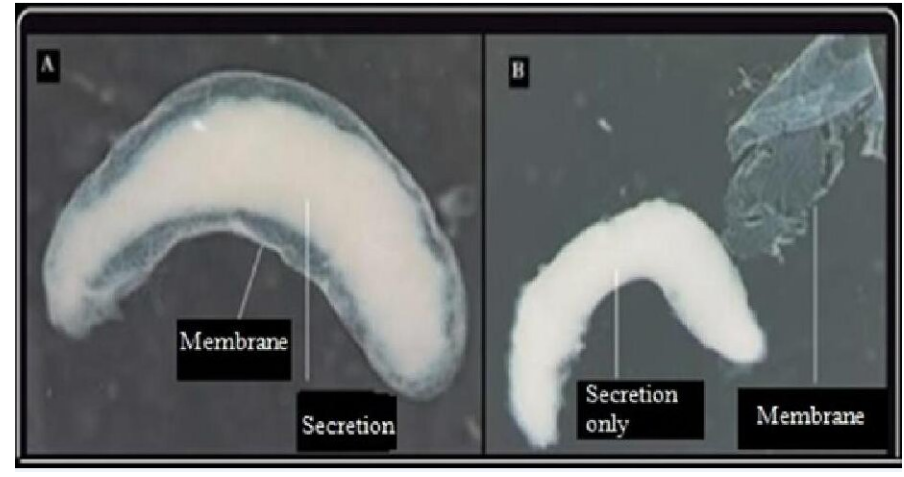

Figure 2. Male accessory gland was set with Ethanol and these were utilized for a quantitative measure of protein A. External covering of the gland. B; These external covering are taken for the measure the amount of accessory gland. Various symbols in superior demonstrate significance at 0.005 levels by Analysis of variance along with Tukey's HSD test. Excellent quality picture information is accessible on the internet.

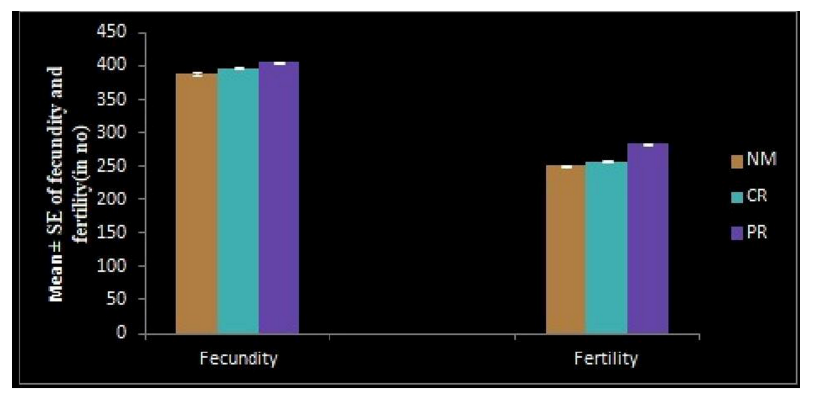

Figure 3. Effect of Male diet on fecundity and Fertility in Drosophila melanogaster.

\subsection{Relation among male diet and amount of entire ac- cessory gland proteins in males which is not mated}

Unmated male sample preparation: Unmated (etherized) males developed from different dietary medium (NM, CR, PR medium) were used to obtain secretion of the gland (Figure 2). Using methanol and chloroform, the secretion was washed and made it to dry at $37^{\circ} \mathrm{C}$ in an incubator for 15 min. To dissolve the accessory gland and discharge, $100 \mu \mathrm{l}$ of sample buffer (0.625 Mtris-HCL pH 6.8, $1 \%$ SDS, $1 \%$ bmercaptoethanol, and $10 \%$ glycerol) was supplementary to all samples to break up the glands and secretions. 10 pair accessory gland were separately collected from each of three diets (normal, carbohydrate-rich and protein-rich media) for significant evaluation of Acps utilized Lowry's methodology [14].

\subsection{Significant evaluation of accessory gland protein uti- lizing Lowry's method}

$50 \mu l$ of Acp was collected from both unmated and mated males. The accessory gland protein substance in the homogenate was set by Lowry's methodology utilizing BSA (Sigma chemicals, St.Louis, MO, USA) as the standard [14]. A total of 50 trials were processed separately for unmated/mated males. A separate experiment was run for each of the NM, CR,
PR media developed flies.

2.4 Connections among male diet, a period of copulation, Amount of Acp in copulated males and transferred amount of accessory gland proteins and sperm

Preparation of Section in copulated males: To quantify Acps of copulated males, five days old virgin female raised in normal diet along with 5 days old unmated males (grown in $\mathrm{NM} / \mathrm{CR} / \mathrm{PR}$ ) were individually situated into an Elens-Wattiaux mating chamber [15] these were noticed for an hour. The pair whichever is not mated inside within an hour were rejected and throw out. In case of copulation happened, the timing of copulation was noted (period among starting to the extinction of copulation of each pair). Right away after copulation (within 5 $\mathrm{min}$ ), Accessory gland protein was obtained from mated males and procedure was done as described above in obtaining Acp of unmated males.

\section{Statistical Analysis}

One way analysis of variance followed by Tukey's HSD test was done for a span of copulation, Accessory gland protein (number and size of the main cell, size of the accessory gland, Amount of Accessory gland protein, transferred amount of Acps) and sperm traits (spermathecae, seminal receptacle, total sperm transferred) All the above information was done by using Statistical Product and Service Solutions- 20 programs.

\section{Results}

Table 1 indicates the mean number of the size and the number of main cells in the accessory gland, and the amount of protein in males that are not mated grown in NM/CR/PR diet. The highest number of the main cells was observed in males PR diet and least in males reared on the CR diet. Males that are raised on PR-diet have a larger size of main cells in the gland, however more or less the same dimension of main cells in the accessory gland was noticed in males fed with CR/NM diet. Big Size of the accessory gland was seen in males reared on PR, whereas accessory gland size was more or less the same in males reared on CR/NM diet. A further quantity of accessory gland secretion was highest in males reared on PR and least in males reared on the NM diet. One way analysis of variance followed by Tukey's HSD test was done on top of the information indicating the major difference in these traits between diets. Statistical analysis (Tukey's HSD test) indicates that the mean value of the main cells in the gland was found to be significantly larger in males fed on the PR diet than those males reared on CR/NM diet. Similarly, the mean value in the main cells of the accessory glands and accessory gland size was found as more considerably bigger in males fed on PR than those males fed on CR/NM diet as shown by Tukey's HSD test.

Table 2 shows the interaction between copulation duration, amount of Acps, sperm transmitted to females copulated with males reared on different diets in D.melanogaster. Males 
Table 1. Connection among male diet and the size of accessory gland, main cells number and main cells size in Drosophila melanogaster

\begin{tabular}{|c|c|c|c|c|c|}
\hline Parameter & df & $\begin{array}{c}\text { Normal media } \\
(\text { mean } \pm \text { SE })\end{array}$ & $\begin{array}{c}\text { Corbohydrate medium } \\
(\text { mean } \pm \text { SE })\end{array}$ & $\begin{array}{l}\text { Protein medium } \\
\quad(\text { mean } \pm \text { SE })\end{array}$ & F-value \\
\hline $\begin{array}{l}\text { Number of Accessory } \\
\text { Gland cells (Unmated) }\end{array}$ & 150 & $1487 \pm 36.3^{a}$ & $1391.60 \pm 36.3^{a}$ & $1566.40 \pm 40.7^{a}$ & $8.364^{\star \star}$ \\
\hline Accessory Gland cell size (mm) & 150 & $0.0669 \pm 0.000009^{c}$ & $0.0066 \pm 0.000009^{c}$ & $0.000075 \pm 0.000001^{c}$ & 0.498 \\
\hline Accessory Gland size $(\mathrm{cm} 2)$ & 150 & $0.3357 \pm 0.006^{c}$ & $0.3350 \pm 0.0005^{c}$ & $0.339 \pm 0.0004^{c}$ & $17.941^{\star \star}$ \\
\hline $\begin{array}{l}\text { Quantity of Protein in } \\
\text { Unmated males (mg/male) }\end{array}$ & 150 & $15.986 \pm 0.043^{c}$ & $15.8020 \pm 0.035^{a}$ & $15.9880 \pm 0.0449^{a}$ & $6.516^{\star \star}$ \\
\hline
\end{tabular}

reared on PR diet copulated longer and transferred a larger amount of sperm and Acps to mated females than individual males raised on CR/NM diet. One way analysis of variance followed by Tukey's HSD test indicates significant difference on top of a character among males reared on different diets. After the result of Statistical test (Tukey's Post Hocof Oneway Anova) it has been noticed that the copulation period, the protein quantity and sperm transmitted indicate that the span of coupling and the amount of protein and sperm transmitted to copulated females were significantly higher in males reared on PR diet than those males reared on CR/NM diet. Furthermore, Tukey's HSD test, It was found that the copulation period, the quantity of protein,sperms moved to females which are mated are notably low in male fed on NM diet than in males fed on the CR diet. The amount of Acps was significantly more in males raised on PR than those flies reared on CR and NM mated males reared on PR was significantly greater than mated males reared on CR and NM diets. Correspondingly, Tukey's HSD test indicated that the amount of the accessory gland, the protein was remarkably prominent in males reared on CR diet than males reared on the NM diet.

Table 2 also shows the relationship among male diet on the copulation duration, Amount of accessory gland protein in mated males, amount of accessory gland protein moved to mated female, reproductive condition (fecundity and fertility) of D. melanogaster are revealed in the graph (Figure 3). Similarity it was seen that the copulation period, the protein quantity which is transferred to females, fecundity and fertility were significantly greater in males reared on PR diet compared to males reared in CR/NM diets. One way analysis of variance (Tukey's HSD) the test indicated that males raised on NM diet have a considerably less copulation period and transmitted less amount of Acps and also noticed that significantly less in fecundity and fertility when compare to the individuals who copulated among males reared on PR/CR diets. Furthermore, males reared on $\mathrm{CR}$ diet shows less significant on copulation period and transmitted less amount of Acp to the mated females, had essentially shown lesser fecundity and fertility than the individual who copulated with males raised on PR diet while indicated in Tukey's HSD test. Males which are reared on the PR diet possess significantly larger protein quantity after contrasted with males raised on CR/NM diet.

\section{Discussions}

The main food nutrients of Drosophila are protein and carbohydrate they feed these from fruits, Quantity, and availability of these nutrients varies between fruits they eat. Careful observation of Table $1 \& 2$ shows that males reared on a protein-rich diet have higher amount of protein synthesis and more number of a smaller main cell exist in glands, whereas males raised on carbohydrates rich diet shows low production of protein synthesis because it has fewer number of bigger main cells. Therefore, a survey in D.melanogaster implies that the number and the size of the main cell which exists in the accessory gland and gland size take part in an essential role in the synthesis of Acps. Quantity of nutrients has a major influence on the size and the number of the main cell in the accessory gland was responsible for the amount of Acps. Additionally while surveying in $D$. nasuta have also Ravi Ram and Ramesh (2002) purpose that amount of Acps might depend on the secretary activity of the main cell in the gland of accessory [5].

Both intrinsic and extrinsic factors are known to affects the amount of Accessory gland protein in species of Drosophila [5-16]. Quantity of Acp varies between species between strains of the same species [16] suggesting that secretion of accessory gland proteins control by both genetic and environmental factors. Ningegowda and Ramesh [6] who while studying in various strains of D.melanogaster and D. nasuta, have found that body size also influences the amount of Acps. They indicate that a greater amount of Acps was seen in larger males than those of smaller males. likewise, age-linked changes in the amount of Acps shown in various species of Drosophila [11-10]. In D.melanogaster, the middle and old age flies indicate a significantly lower quantity of Acps, wherein younger males show higher significance in the amount of Acps [4]. In comparison to these flies of $D$. bipectinata protein fed older males show a significantly better amount of accessory gland protein than the young and mid-aged flies [11] signifying that age of the male had a significant influence on the quantity of accessory gland in Drosophila species. The accessory gland of D.melanogaster contains main cells which are involved in the creation of accessory gland, therefore, it is presumed that variation in the amount of Acps in unmated males reared on various diet possibly be due to the difference in the size of the accessory gland or number/cell size of the main cell in the accessory gland or the difference in the secretary activity of the accessory gland with the male diet regime.

Furthermore, the synthesis of Acp in D.melanogaster was developmentally regulated (Monsma et.al., 1990). In contrast to this outcome, Ravi Ram [5] when he was surveying in D.nasuta had noticed that a less influence of the main cell size of the accessory gland on the amount of protein production. Thus these studies imply that amount of accessory gland protein in unmated not only depends upon the number and the size of the 
Table 2. Estimation of Copulating period was taken minutes, number of sperms are counted and Amount of Acps in $\mu g / \mathrm{male}$ or $\mu \mathrm{g} / \mathrm{pair}$ of glands, *Significant at 0.05 level $(\mathrm{P}<0.05)$; **Significant at 0.001 level $(\mathrm{P}<0.001)$. Various symbol in superior demonstrate significance at 0.005 levels by Analysis of variance along with Tukey's HSD test. Amount of gland protein transmitted was determined through deducting the amount of protein of mated males from unmated males.

\begin{tabular}{|l|c|c|c|c|c|}
\hline \multicolumn{1}{|c|}{ Parameter } & df & $\begin{array}{c}\text { Normal media } \\
(\mathbf{m e a n} \pm \mathbf{S E})\end{array}$ & $\begin{array}{c}\text { Corbohydrate medium } \\
(\mathbf{m e a n} \pm \mathbf{S E})\end{array}$ & $\begin{array}{c}\text { Protein medium } \\
(\mathbf{m e a n} \pm \mathbf{S E})\end{array}$ & F-value \\
\hline Copulation duration (min) & 150 & $17.688 \pm 0.622^{b}$ & $17.3284 \pm 0.4322^{a}$ & $17.8574 \pm 0.4322^{c}$ & $29.833^{\star \star}$ \\
\hline $\begin{array}{l}\text { Amount of Protein in } \\
\text { mated males }(\mu \mathrm{g} / \text { male })\end{array}$ & 150 & $12.030 \pm 0.01750^{b}$ & $12.0060 \pm 0.1166^{a}$ & $12.1180 \pm 0.216^{c}$ & $11.477^{\star \star}$ \\
\hline $\begin{array}{l}\text { Amount of protein } \\
\text { transferred to mated female }\end{array}$ & 150 & $6.545 \pm 0.0258^{a}$ & $6.6684 \pm 0.0315^{c}$ & $6.5456 \pm 0.2580^{b}$ & $1894.81^{\star \star}$ \\
\hline Spermthecae & 150 & $33.82 \pm 0.369^{a}$ & $22.44 \pm 0.347^{b}$ & $16.12 \pm 0.554^{b}$ & $49.366^{\star \star}$ \\
\hline Seminal receptacle & 150 & $173.66 \pm 4.788^{a}$ & $108.72 \pm 0.713^{b}$ & $88.64 \pm 0.842^{c}$ & $184.22^{\star \star}$ \\
\hline Total sperm transferred & 150 & $6431.9 \pm 3.570^{a}$ & $4709.14 \pm 2.79^{b}$ & $3282.4 \pm 10.3^{c}$ & $1617.2^{\star \star}$ \\
\hline
\end{tabular}

main cell in the accessory gland but also depends on the secretary activities of these cells and age [17]. In the current study flies of the same age were used and conditions for maintenance were also the same only difference was the nutrients such as protein and carbohydrate. Therefore observed variation in the quantity of Acps was due to the influence of male diet on the number, size, and secretary activity of main cells in the accessory gland in D.melanogaster.

In the current survey, unchanged pair of flies used to examine copulation were also used to measure accessory gland protein and sperm to understand the interrelationship between diet, copulation duration, and amount of accessory gland proteins transferred to mated females. Table 2 shows that male reared on the protein-rich diet had copulated significantly longer and transmitted a larger amount of accessory gland proteins and sperm to mated females than those males reared on CR or NM diet.

This outcome effect described by the following hypotheses.

1) Males reared on the protein-rich medium were not possibly able to transferred sperm rapidly and hence require longer copulation.

2) Males reared on the protein-rich medium may transfer larger quantities of sperm to mated females, therefore they need more time (Table 2).

3) Males reared on protein-rich medium, may also suggest that males reared on protein-rich medium provide more resource per mating.

Thus in D.melanogaster males reared on protein-rich medium with longer copulation duration had transmitted a larger amount of protein and sperm to the mated females, this confirms the $2^{\text {nd }}$ and $3^{\text {rd }}$ hypothetical explanation. Males reared on carbohydrate-rich medium had transmitted low protein and sperm to copulated females. If sperm are indeed costly, the diet should have an effect on the number and/ or the potential of sperm produced. Diet also influences sperm synthesis in several mammalian species [18], including humans [19].

Though, in some surveys, it has analyzed that the affect of the male diet also influences sperm synthesis in insects. Moreover, very few surveys that have analyzed this connection in insects have yielded equivocal results. Queensland, fruit flies [20], D.melanogaster [21], red flour beetles [22], and Indian meal both [23] show an increase in sperm production on higher quantity diets. Thus this study in D.melanogaster male nutrition has a significant influence on male reproductive success such as accessory gland and sperm traits.

\section{Conclusions}

Based on the findings of this research, Drosophila melanogaster male reproductive success changes according to the quality of the available diet, which are correlated with reproductive adjustment at structural and functional levels.

\section{Acknowledgements}

The author would like to thank UGC under RGNF for supporting financially. Letter number. F1-17.1/RGNF2015-17-SC-KAR-18572/(SA-III/Website), Zoology department, Mysore university, Manasagangothri, Mysuru, Karnataka, India.

\section{REFERENCES}

[1] Nothiger, R., Dubendorfer, A., Epper, F. (1977) Gynandromorphs reveal two separate primordial for male and female genitalia in Drosophila melanogaster. Devel Biol 181: 367-373.

[2] Bairati, Aurelio.(1966) Filamentous structures in spermatic fluid of Drosophila melanogaster Meig. J. Microscopie, 5: 265265.

[3] Wolfner, M.F. (1997) Tokens of love: functions and regulation of Drosophila male accessory gland products. Insect Biochem and Mol Biol 27: 339-346.

[4] Chen, P.S. (1984) The functional morphology and biochemistry of insect male accessory glands and their secretions. Ann rev of ent 29: 233-255.

[5] Ravi Ram, K., Ramesh, S.R. (2002) Male accessory gland secretory proteins in nasuta subgroup of Drosophila: Synthetic Activity of Acp. Zool Sci 19: 513-518.

[6] Ningegowda, N.L., Ramesh, S.R. (2004). Male accessory glands in Drosophila: a study on relationship between quantity of secretory proteins and body size. Entomon 29(1): 57-62. 
[7] Friberg, U. (2006) Male perception of female mating status: its effect on copulation duration, sperm defence and female fitness. Anim Behav 72: 1259-1268.

[8] Wigby, S., Domanitskaya, E.V., Choffat, Y., Kubli, E., Chapman, T. (2008) The effect of mating on immunity can be masked by experimental piercing in female Drosophila melanogaster. J of Insect Phys 54: 414-420.

[9] Bretman, A., Fricke, C., Chapman, T. (2009) Plastic responses of male Drosophila melanogaster to the level of sperm competition increase male reproductive fitness. Proc of the R Soc B: Biol Sci 297(1662): 1705-171.

[10] Abolhasan Rezaei, Mysore Siddaiah Krishna, and Hassan T. Santhosh. (2015) Male Age Affects Female Mate Preference, Quantity of Accessory Gland Proteins, and Sperm Traits and Female Fitness in D. melanogaster. Zool Sci 32: 16-24.

[11] Santhosh, H.T. and Krishna, M.S. (2013) Relationship between male age, accessory gland, sperm transferred, and fitness traits in Drosophila bipectinata. J. Insect Sci 13 (1): 159.

[12] Delcour, J. (1969) A rapid and efficient method of egg collecting. Drosophila Inform Serv 44: 133-134.

[13] Ashburner, M. (1970) Patterns of puffing activity in the salivary gland chromosomes of Drosophila. V. Responses to environmental treatments. Chrom 31: 356-76.

[14] Lowry, O.H., Rosebrough, N.J., Farr, A.L., Randall, R.J. (1951) Protein measurement with the Folin phenol reagent. J Biol Chem 193:265-275.

[15] Elens, A.A., Wattiaux, J.M. (1964) Direct observation of sexual isolation. Drosophila Inform Serv 39: 118-119.
[16] Shivanna, N. and Ramesh, S. R. (1995) Quantitative and qualitative analysis of accessory gland secretory proteins in a few species of Drosophila immigrans group. Ind. J. Exp. Biol. 33: 668-672.

[17] Monsma, S.A., Harada, H.A., Wolfner, M.F. (1990) Synthesis of two Drosophila male accessory gland proteins and their fate after transfer to the female during mating. Devel Biol. 142: 465475 .

[18] Coulter, G., Cook, R., Kastelic, J. (1997) Effects of dietary energy on scrotal surface temperature, seminal quality, and sperm production in young beef bulls. J. Anim. Sci. 75: 1048-1052.

[19] Olsen, J., Ramlau-Hansen, C.H. (2012) Dietary fats may impact semen quantity and quality. Asian J. Androl. 14: 511-512.

[20] Perez-Staples, D., Harmer, A.M., Collins, S.R., Taylor, P.W. (2008) Potential for pre-release diet supplements to increase the sexual performance and longevity of male Queensland fruit flies. Agric. Forest Entomol. 10: 255-262.

[21] McGraw, L.A., Fiumera, A.C., Ramakrishnan, M., Madhavarapu, S., Clark, A.G., Wolfner, M.F. (2007) Larval rearing environment affects several post-copulatory traits in Drosophila melanogaster. Biol. Lett. 3: 607-610.

[22] Fedina, T.Y., Lewis, S.M. (2006) Proximal traits and mechanisms for biasing paternity in the red flour beetle Tribolium castaneum (Coleoptera: Tenebrionidae). Behav. Ecol. Sociobiol. 60: 844-853.

[23] Gage, M.J.G., Cook, A.P.(1994) Sperm size or numbers? Effects of nutritional stress upon eupyrene and apyrene sperm production strategies in the moth Plodia interpunctella (Lepidoptera: Pyralidae). Funct. Ecol. 8: 594-599. 